\title{
Ambulante Tarifrevision: Projekt TARCO - wir bleiben dran!
}

\section{Urs Stoffel}

Dr. med., Mitglied des FMH-Zentralvorstandes, Departementsverantwortlicher Ambulante Versorgung und Tarife

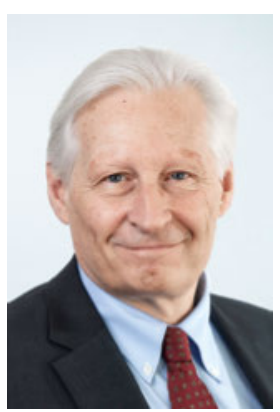

Am 7. September 2016 hat die Delegiertenversammlung der FMH (DV) dem Zentralvorstand, nach der Ablehnung des Tarifvorschlags durch die Urabstimmung, wieder ein Mandat für die Weiterführung der Tarifrevision erteilt und das Grobkonzept zum Tarifrevisionsprojekt TARCO genehmigt. Das Departement Ambulante Versorgung und Tarife wurde beauftragt, ein Detailkonzept auszuarbeiten, das der DV im Januar 2017 zur Genehmigung vorgelegt werden soll. Das Projekt wird dabei auf den bereits geleisteten Arbeiten aufbauen und strebt Nachbesserungen vor allem zur Stärkung der ärztlichen Leistung gegenüber den technischen Infrastrukturund nichtärztlichen Personalleistungen an.

\section{Die Tarifrevision soll weitergehen}

Seit dem negativen Urabstimmungsentscheid der FMH, der Ablehnung der Tarifstruktur durch die Kostenträger und dem ungenutzten Verstreichen der vom Bundesrat gesetzten Nachfrist vom 31. Oktober 2016 hört man von verschiedenen Seiten, dass die Tarifrevision hoffnungslos blockiert und gescheitert sei und auf absehbare Zeit keine tarifpartnerschaftliche Lösung zu erwarten ist. Das Gegenteil ist der Fall: Das Departement Ambulante Versorgung und Tarife der FMH ist mit Hochdruck daran, unter Einbezug der Dach- und Fachgesellschaften ein konsensfähiges Detailkonzept für die Weiterführung der Tarifrevision auszuarbeiten. Schon am 1. Juli 2016 hat die FMH gegenüber dem BAG und der

\section{Die FMH arbeitet mit Hochdruck an der Weiter- führung der Tarifrevision.}

Öffentlichkeit kommuniziert, dass sie weiterhin an einer Tarifrevision festhält und dass sie die Einreichung eines gemeinsamen tarifpartnerschaftlichen Vorschlags weiterverfolgen will.

Allerdings ist es unrealistisch, einen solchen sowohl FMH-intern wie auch bei den Tarifpartnern breit abgestützten revidierten Tarifvorschlag zu erarbeiten, nur vier Monate nach der Ablehnung durch die Urabstimmung. Obwohl inzwischen der Bundesrat einen Tarif- eingriff in die jetzige Tarifstruktur für 2018 angekündigt hat, sind wir der Meinung, dass weitere punktuelle Eingriffe in die bestehende, nicht mehr sachgerechte und nicht mehr betriebswirtschaftliche Tarifstruktur zu einer weiteren Verzerrung des Tarifs führen.

Eine Tarifrevision unter Einbezug aller Ärzteorganisationen und Tarifpartner ist für die FMH der einzig richtige Weg.

Die FMH schlägt deshalb einen anderen Weg ein und hat aus dem abgelehnten Projekt TARVISION die nötigen Lehren gezogen. Zurzeit sind wir intensiv daran, die tarifübergreifenden «Stolpersteine» aus dem Weg zu räumen. Der Umgang mit den qualitativen und quantitativen Dignitäten und auch die Tarifierung der Leistungen rund um den OP-Bereich müssen in den paritätisch zusammengesetzten Arbeitsgruppen aus Vertretern der Dachverbände - bereits vor dem eigentlichen Start des Projekts TARCO - verbindlich festgelegt und geregelt werden.

Ein weiterer Fokus des Revisionsprojekts liegt darin, die interne Kommunikation zu den Fach- und Dachgesellschaften und zur Basis der FMH zu verbessern. Nur durch eine intensive und transparente Kommunikation zur Basis kann Vertrauen und Akzeptanz für das Projekt TARCO geschaffen werden.

\section{Die nächsten Meilensteine sind gesetzt}

Unmittelbar nach der Genehmigung des Detailkonzepts Ende Januar 2017 durch die DV starten die Arbeitsgruppen unter Einbezug der Fachgesellschaften die Nachbesserungen in den einzelnen Tarifkapiteln. Bis Ende 2017 soll ein FMH-interner und konsensfähiger Vorschlag auf dem Tisch liegen, der dann mit den Tarifpartnern abgestimmt und finalisiert werden soll. Ein gemeinsamer Vorschlag könnte so von den Leistungserbringern und den Kostenträgern Mitte 2018 dem Bundesrat zur Genehmigung eingereicht werden. Deshalb ist für die FMH eine umfassende Tarifrevision unter Einbezug aller Ärzteorganisationen und Tarifpartner der einzig richtige Weg. 